\title{
Effects of damping on the speed of increase and amplitude of limit cycle for an aircraft braking system subjected to mode-coupling instability
}

\author{
F. Chevillot ${ }^{1,2}$, J.-J. Sinou ${ }^{1}$, N. Hardouin ${ }^{2}$ and L. Jézéquel ${ }^{1}$ \\ ${ }^{1}$ Laboratoire de Tribologie et Dynamique des Systèmes UMR-CNRS 5513 \\ Ecole Centrale de Lyon, 36 avenue Guy de Collongue, 69134 Ecully Cedex, France \\ ${ }^{2}$ Messier-Bugatti, Aircraft Braking Division \\ Zone Aéronautique Louis Bréguet, BP 40, 78140 Vélizy-Villacoublay, France
}

\begin{abstract}
A nonlinear model of an aircraft braking system is presented and used to investigate the effects of damping on the stability in Chevillot et al. (Arch Appl Mech 78(12):949-963, 2008). It has been shown that the addition of damping into the equations of motion does not lead systematically to the stabilization of the system. In the case of a mode-coupling instability, there is indeed an optimal ratio between the modal damping coefficients of the two modes in coalescence, that maximize the stable area. But the stable area is not a sufficient criterion. In dynamics, the amplitude of the vibrations and the transient behavior characterized by the speed of increase of the oscillations are best indicators. In this paper, the same nonlinear model of the aircraft braking system is used to compute time-history responses by integration of the full set of the nonlinear dynamic equations. The aim of the study is to evaluate the effects of damping on the nonlinear dynamics of the brake. It is shown that damping may be very efficient to significantly reduce and slow down the increase of the friction-induced vibrations. But, in the same way as for the stability area, there exists a value of the damping ratio that optimizes the effects of damping.
\end{abstract}

Keywords: friction-induced vibration, damping, limit cycles, nonlinear dynamics

\section{Introduction}

The problem of unstable vibrations in mechanical systems with rotating and sliding parts has received the attention of many investigations, and is still and active field of research in dynamics. Solving potential vibration problems requires the consideration of the stability analysis and the determination of the time-history response. A stability analysis as presented in The destabilization paradox applied to friction-induced vibrations in an aircraft braking system [1] allows determining the characteristics (frequency, real part, mode shape and critical friction coefficient) of the instabilities that may appear during the braking by extraction of the eigenvalues of the linearized system at the equilibrium point. In particular it allows knowing if for a given set of parameters the system is stable or not. But if not, it gives no information on the amplitude of the oscillations or on the nonlinear transient behavior. To achieve this, the complete nonlinear response of the system has to be computed. 
In this paper, the model developed in [1] is used to investigate the effects on damping on the nonlinear dynamical behavior of an aircraft braking system. The results of [1] indicate that the addition of damping in the equations of motion alter the stable-unstable boundary. In some cases, a destabilizing effect was shown: damping is not always beneficial. The paper also shows that for this particular system there exists an optimal structural damping ratio between the stable and unstable modes for which the stable region is the largest.

The effects of damping on non-linear non-conservative systems and the highlight of a destabilizing paradox was first studied by Ziegler [2]. The study was focused on a double pendulum under a follower force. He showed that the critical load of the damped system was significantly smaller than that of the undamped system. The fact that damping can be harmful to a system is not intuitive and runs counter to the generally accepted idea of the effects of damping. Many investigators have subsequently looked into this phenomenon. In particular, Bolotin [3] showed that the ratio between the damping coefficients plays a key role in the destabilizing effect. Later on, Herrmann [8], Walker [9], O'Reilly et al. [6] and recently Kirillov and Seyranian $[7,11]$ worked on the determination in explicit form of the stabilizing damping configurations for a large class of non-conservative systems. In particular in [7] necessary and sufficient conditions for the matrices of velocity-dependent forces to stabilize an unperturbed circulatory system have been expressed. Applied to the Ziegler pendulum, it was found that the optimum damping ratio that gives the larger critical load is far from unity. In wood cutting machines, Gallina and Trevisani [10] demonstrated that similar damping coefficients cannot destabilize the initially stable system. Using a finite element model Fritz [13] came to the same conclusion for an automotive brake squeal model. An asymmetry in the damping distribution tends indeed to make the system unstable for a lower value of a the friction coefficient. However, Sinou and Jézéquel [12] for example studied the effects of damping with a nonlinear two-degree-of-freedom model with friction and found that an optimal damping distribution not always exists. The damping coefficients, their ratio, and the pulsation ratio have to be considered. Hervé et al. [14] used a two-degree-of-freedom nonlinear model of clutch squeal to investigate the influence on circulatory and gyroscopic actions. While for the purely circulatory system proportional damping is found to be the optimum, taking into account the gyroscopic effects makes a lot more complex the effects of damping. Moreover, most of these studies focused on the effects on the stability region. However, because a stability analysis is based on the nature of the eigenvalues of the linearized equations of motion, it is not obvious that the effects on the nonlinear system are the same. Aware of that, recent papers (Kounadis [5], Bolotin et al. [4], Sinou and Jézéquel [12] for instance) included in their studies the analysis of the steady-state amplitudes. It is motivated by finding that regions considered unstable by a stability analysis turn out to be stable when a non-linear dynamic analysis is performed (Kounadis [5]).

In this paper, the model developed in [1] is briefly exposed. Then, the analysis of the stability of the system is presented. While Chevillot et al. [1] deal with three low-frequency mode-coupling instabilities calculated, this study focuses on the second instability, for which the evolution of the stability area versus the friction coefficient and the damping ratio is reminded. Time-history simulations with variations in the friction coefficient and damping configuration are performed to analyze their effects on the transient behavior and the limit cycle amplitude of the instability. The main purpose of the present paper is to find if the conclusions expressed in [1] about the stability regions are similar when a nonlinear dynamic analysis is performed. The results show that damping is a key factor very efficient to reduce frictioninduced vibrations, but has to be used cautiously to avoid misuse. In particular, the destabilizing effect and the optimum damping distribution highlighted in [1] for the stability analysis are extended to the nonlinear 
dynamics of the system.

\section{Overview}

\subsection{Model description}

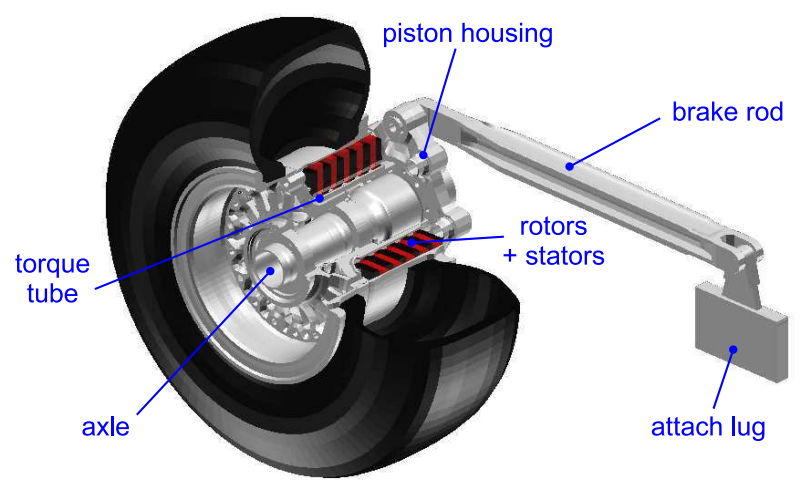

(a) Aircraft braking system

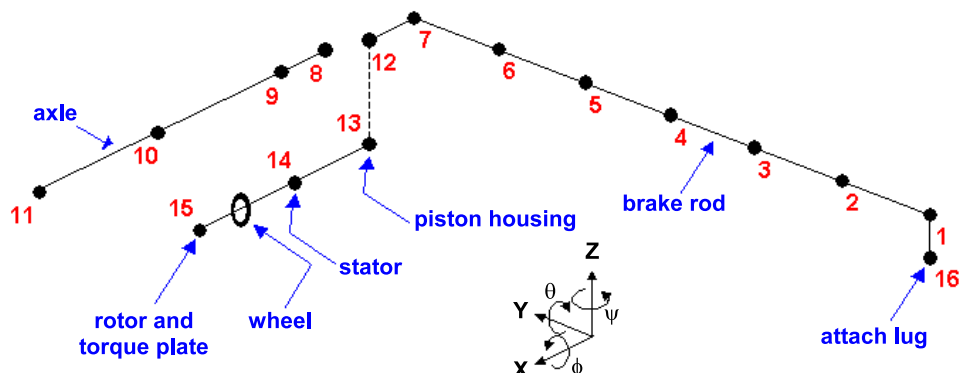

(b) Parametric model of the brake

Figure 1: Aircraft braking system description

Based on experimental observations, an analytical model of an aircraft braking system given in Fig. 1a has been developed and presented in [1] in order to reproduce whirl and squeal instabilities. The view of the parametric model using 70 degrees of freedom in Fig. 1b allows seeing the correspondence between the model and the system. The multi-stage brake is represented by a single rotor and stator with an effective brake friction coefficient of $\mu_{e q}=2 n \mu$ where $n$ is the number of rotors, producing $2 n$ interfaces in contact between the stators and rotors. It is assumed that the friction coefficient is uniform along the rubbing surface and that the rotor and stator friction surfaces are always in contact. Friction is modeled by a Coulomb approach: the tangential force is related to the normal force by the friction coefficient (for more details see [1]). Experimental tests show that the behavior of the rotor/stator stack in compression is nonlinear. The analytical model uses a third order polynomial to express the load-deflection relationship [1].

The global nonlinear forces and moments due to friction are then expressed (see [1]), and the hydraulic pressure, tire-ground drag force and wheel bending load are introduced. By considering the interconnections between each element of the braking system, the equations of motions take the form:

$$
\mathbf{M} \ddot{\mathbf{x}}+\mathbf{C} \dot{\mathbf{x}}+\mathbf{K x}=\mathbf{F}_{\text {contact }}+\mathbf{F}_{\text {hydr }}+\mathbf{F}_{\text {drag }}+\mathbf{F}_{\text {load }}
$$

where $\ddot{\mathbf{x}}, \dot{\mathbf{x}}$ and $\mathbf{x}$ are respectively the 70 -dimensional vectors of acceleration, velocity and displacement. $\mathbf{F}_{h y d r}$ is the vector force due to brake hydraulic pressure, $\mathbf{F}_{\text {contact }}$ contains the linear and nonlinear contact force terms at the stator and rotor interface, $\mathbf{F}_{\text {drag }}$ represents the tire-ground drag force, and $\mathbf{F}_{\text {load }}$ the bending load of the wheel. $\mathbf{M}, \mathbf{C}$ and $\mathbf{K}$ are respectively the structural mass, global damping and stiffness matrices. 


\subsection{Stability analysis}

In a preliminary study, the stability analysis of the brake is performed. It is obtained for a small perturbation around the steady-state operating point by calculating the eigenvalues of the linearized system. The detailed methodology can be found in [1]. For $\lambda$ a complex eigenvalue of the form $\lambda=a+\imath b$, the stability criterion is: if $a$ is negative or zero, the system is stable and no vibration occurs, and if $a$ is positive then the system is unstable. The imaginary part $b$ represents the angular pulsation (or the frequency by considering a factor of $2 \pi$ ) of the vibration. The plot of the angular pulsations versus the brake friction coefficient is given in Fig. 2. The graph is normalized towards the pulsation of the first instability $\omega_{0,1}$ and towards the maximum friction coefficient $\mu_{\max }$ used in the study. The red plots define the appearances of instability.

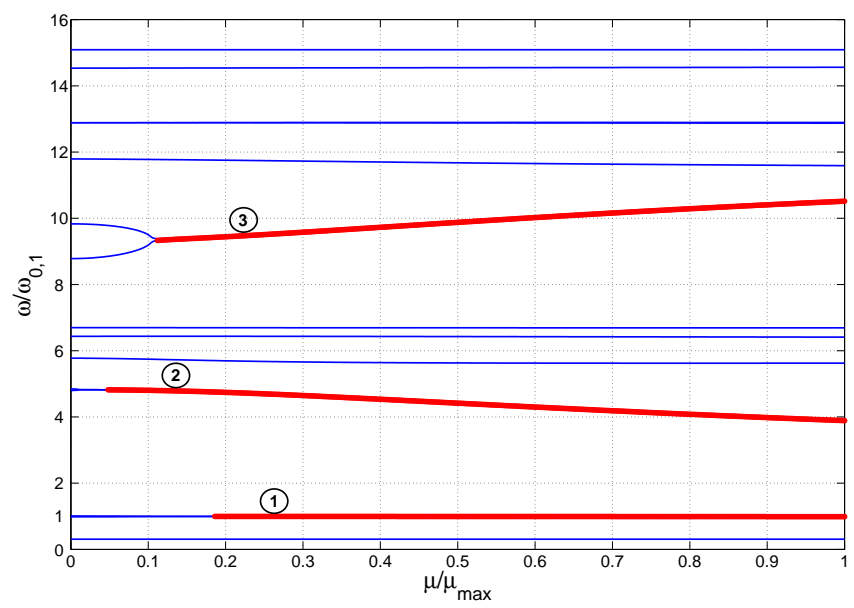

Figure 2: Angular pulsations versus friction coefficient

It appears that six of the fourteen firsts modes are involved in mode-coupling instabilities. The nature of each instability is determined by plotting its analytical shape. The mode shapes of all the instabilities can be found in [1]. The second instability will be the matter of the following study.

In [1] parametric studies are performed to evaluate the effects of damping or other parameters on the stability of the brake. The effects of damping on the second instability are summed up in Fig. 3, where the stability area is given when the damping ratio $\eta_{1} / \eta_{2}$ between the two modes producing the instability varies. It turns out that there exists a value of the damping ratio for which the stability area is the widest. This optimum ratio is near 1 , that means the optimum ratio corresponds to $\eta_{1} \simeq \eta_{2}$. In fact, in [1] it is shown that the optimum damping ratio corresponds to a proportional damping configuration, given by $\eta_{1} / \eta_{2}=\omega_{0,2} / \omega_{0,1}$, where $\omega_{0,1}$ and $\omega_{0,2}$ are the pulsations at $\mu=0$ of the two modes producing the instability. But given that $\omega_{0,1}$ and $\omega_{0,2}$ are very close, the relation can be in first approximation expressed by $\eta_{1} \approx \eta_{2}$, ie for a damping ratio close to 1 . For values of the damping ratio far from the optimum value, the stability may be clearly affected, even leading to a more unstable system that the undamped system! That illustrates the phenomenon of "destabilization paradox" presented in introduction of the paper. 


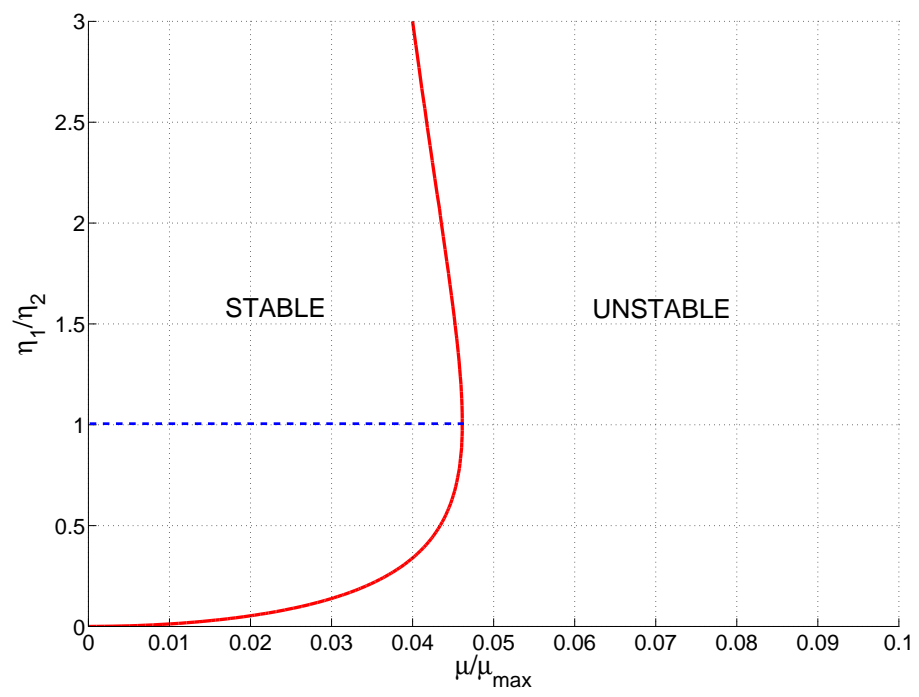

Figure 3: Stability (stable and unstable zones) versus $\mu / \mu_{\max }$ and $\eta_{1} / \eta_{2}$ for the second whirl instability

\section{Nonlinear dynamics}

\subsection{Introduction}

Because damping appears to be a key factor, its effects on the dynamical behavior of the aircraft braking system must be examined. Determining the stable and unstable regions is only one aspect of the problem of friction-induced flutter instability. However, both the maximum amplitude of the instability and the time to reach the limit cycle are more significant design factors than the stability region.

The nonlinear equations of motion (1) can be integrated numerically to obtain time-history responses. For this study the case of the second instability (the second whirl) presented above is considered. For a value of $\mu / \mu_{\max }$ between 0.045 and 0.115 , this is the only instability that can occur (see Fig. 2), so it will simplify the analysis of the effects of parameters on the nonlinear behavior. In Fig. 4a the time-history response of the vertical deflection of the end of the axle is given. The simulation has been made with a friction coefficient $\mu / \mu_{\max }$ of 0.075 and with an arbitrarily chosen damping configuration. One observes two different stages: first an initiation phase (I) where the oscillations increase around the static position to attain the stabilized phase (II) where the oscillations keep a constant amplitude. These two phases are well represented in Fig. 4b which shows the evolution in the coordinates system (displacement,velocity). The phase (I) is characterized by increasing spirals, and the phase (II) is the stationary limit cycle. With the application of a FFT transformation, it is verified that the signal contains only one vibration at a frequency very close of that given by the stability analysis with linear approximation (in a case of a nonlinear resonance, the frequency of the instability may be altered by nonlinear effects).

In order to understand the effects of structural damping on the oscillation amplitude and the time to reach the limit cycle, the next study is divided into two parts. In the first one, the effects of the friction 
coefficient and damping on the transient behavior are investigated. In the second one, the effects on the limit cycle and maximum amplitude are examined.

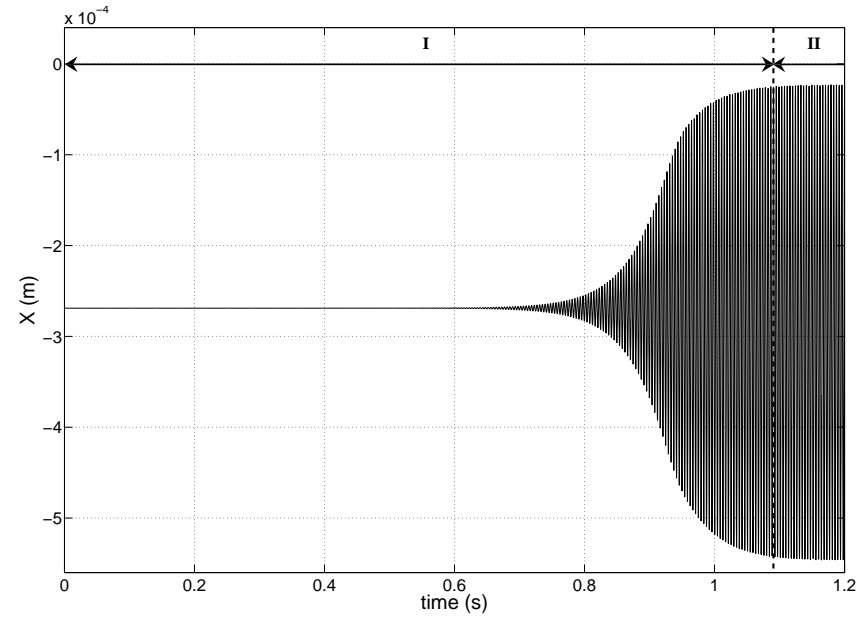

(a) Displacement

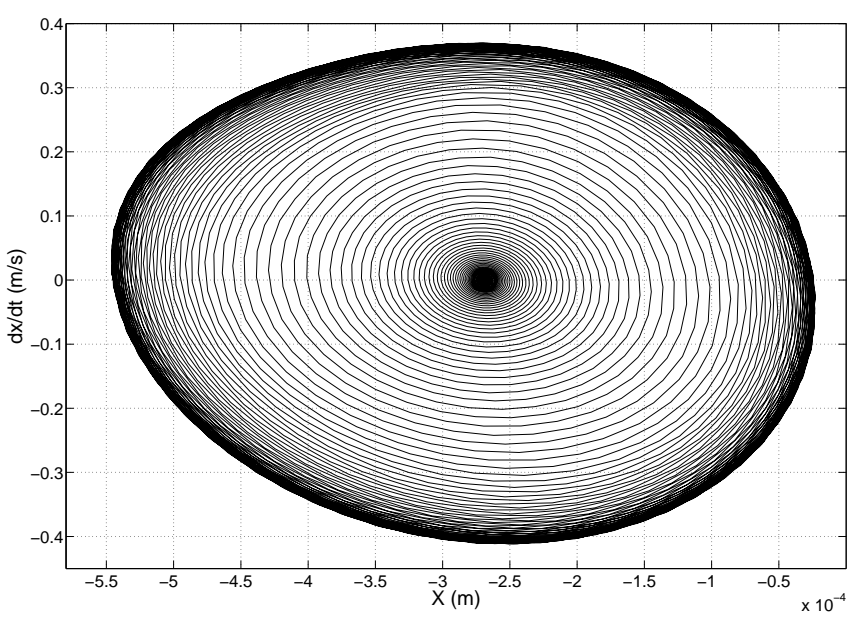

(b) Limit cycle

Figure 4: Vertical displacement of the axle $\left(\mu / \mu_{\max }=0.075\right)$

\subsection{Investigations on the transient behavior}

In this section the transient behavior corresponding to the initiation phase (I) is examined. The characterization of this phase is given by the response time: it represents the time to attain the limit cycle of the vibration. Here a criterion of $98 \%$ of the amplitude of the limit cycle is considered. In Fig. 5 is plotted the response time versus the brake friction coefficient. For a value of $\mu / \mu_{\max }$ under 0.045 , the system is stable and no vibration occurs. It can be seen that in this range of $\mu$ the response time is a decreasing function of the friction coefficient: the closer we are to the bifurcation point (where the two modes couple), the more slowly the vibration appears. But this conclusion requires more investigations. To further our understanding, the inverse of the real part of the mode becoming instable is displayed on the same figure. It is extracted from the eigenvalue analysis: if $\lambda=a+\imath b$ is the eigenvalue with a positive real part, then during the beginning of the phase (I) the displacement can be written in linear approximation by an exponential increase:

$$
\mathbf{x}=\mathbf{A} e^{\lambda t}=\mathbf{A} e^{a t} e^{\imath b t}
$$

where $\mathrm{A}$ is a constant. Thus, the amplitude of the oscillation -of angular pulsation $b$ - is given by $\mathbf{A} e^{a t}$, with a time constant of $1 / a$. Let's note that the stability criterion appears here clearly: if $a$ is positive the argument of the exponential is positive resulting in an increasing oscillation, and if a is negative or zero the amplitude of the oscillation is limited. In phase (II) the nonlinear effects induce the stabilization of the oscillation into the limit cycle. The Fig. 5 shows that a linear relation between the $98 \%$ response time and the inverse of the time constant of the exponential approximation can be established: $t_{98 \%} \approx \frac{20}{a}$. So the results of the stability analysis can be exploited in terms of time-history response, even if the numerical constant in the previous relation seems to be different for each instability. The stability analysis is very quick to obtain for a model 
of only 70 degrees of freedom. Then, in addition of the information obtained on the stability of the system, it also gives an idea of the temporal behavior.

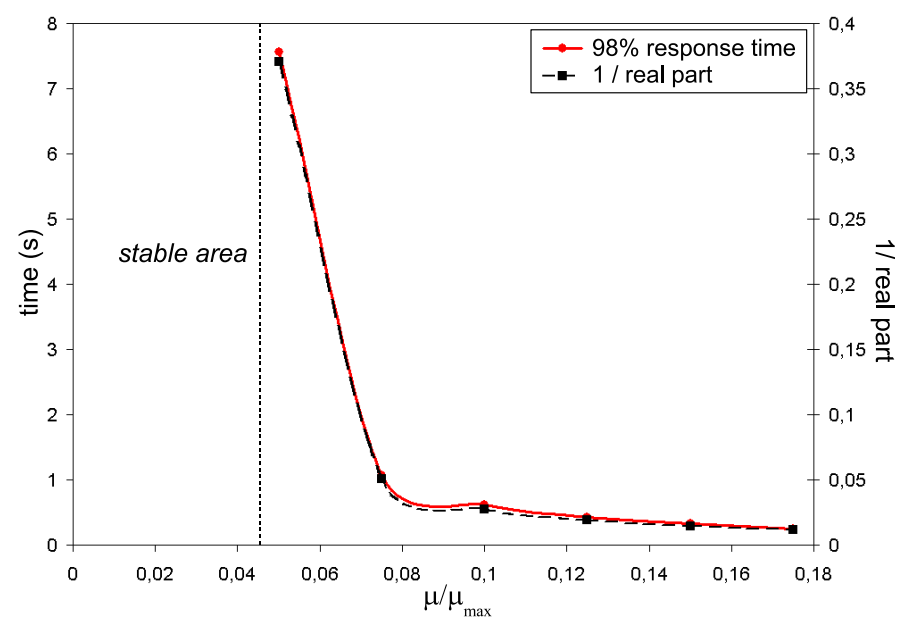

Figure 5: Evolution of the $98 \%$ response time with variations of the friction coefficient

The second point is the evaluation of the effects of damping. Two approaches are undertaken. In the first case, proportional damping is considered: that means that the same damping coefficient $\eta$ is introduced on the two coupling modes of the second whirl. In the second case, non-proportional damping is considered: the values of the damping coefficients $\eta_{1}$ and $\eta_{2}$ vary under the condition $\eta_{1}+\eta_{2}=$ constant. Below are summarized the parameters of the two cases considered, both use a friction coefficient $\mu / \mu_{\max }$ of 0.075 .

\begin{tabular}{|c|c|}
\hline case \#1 & $\eta_{1}=\eta_{2}$, from $0.5 \%$ to $3 \%$ \\
\hline case \#2 & $\eta_{1}+\eta_{2}=4 \%$, with $\eta_{1} / \eta_{2}$ from $1 / 7$ to 7 \\
\hline
\end{tabular}

In the first case corresponding to proportional damping, the analysis is quite simple. Fig. 6a shows that the increase of proportional damping produces the slowdown of the vibration with a longer response time. Thus, from a damping of $0.5 \%$ to $3 \%$, the time the vibration takes to attain the limit cycle goes from 0.8 seconde to 4.1 secondes, ie a ratio of more than 5. In the second case, the presence of an optimum is observed in Fig. 6b: a damping ratio close to 1 pushes back at best the vibration. That coincides with the results of the stability analysis stated in [1] i.e. for this system proportional damping is the optimum configuration. In addition these two studies show once more the good relation between the response time and the real part of the unstable mode.

\subsection{Investigations on the maximum amplitude of oscillations and limit cycle}

This second section deals with the evaluation of the effects of friction coefficient and damping on the limit cycles and maximum amplitudes of oscillation in phase (II).

First, limit cycles are plotted for several friction coefficients. The chosen degrees of freedom are the horizontal and vertical deflections of the end of the axle, and the horizontal and vertical displacements of the middle of the brake rod. They are indeed elements in motion during the occurrence of the second 


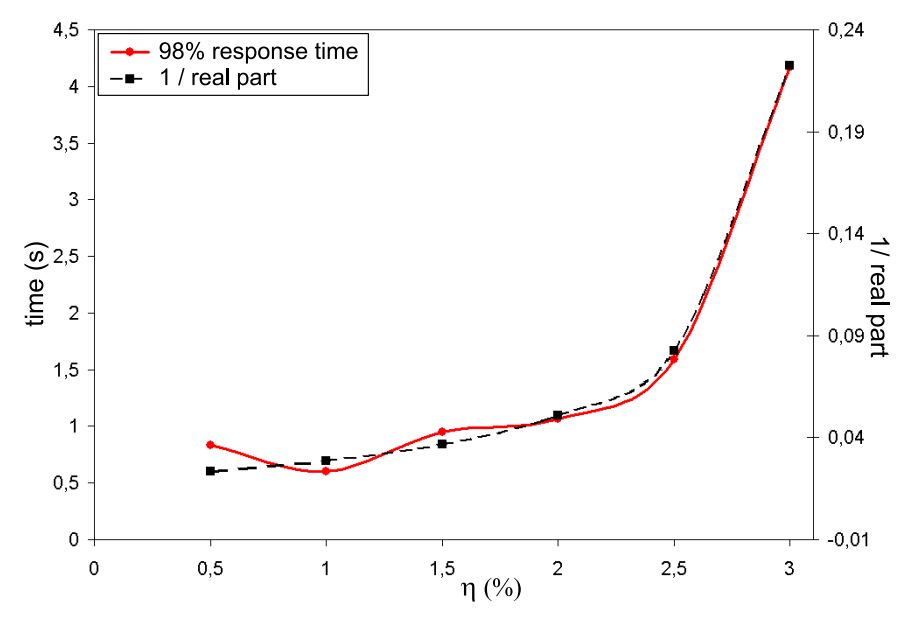

(a) Proportional damping

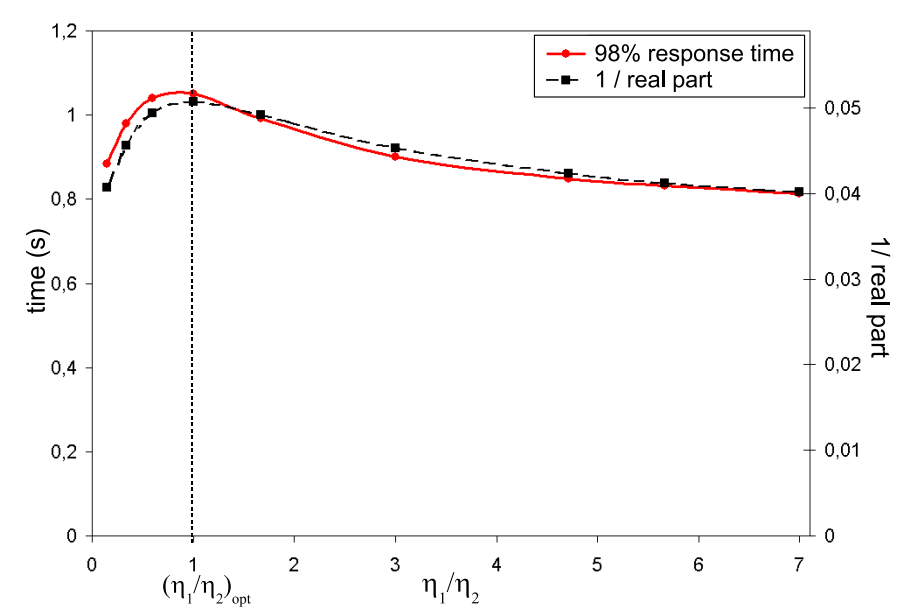

(b) Non-proportional damping

Figure 6: Evolution of the $98 \%$ response time with variations of damping

whirl. It may be observed that on average the increase of the friction coefficient leads to larger limit cycles. So, as expected, when moving away from the bifurcation point by increasing $\mu$, the system becomes more unstable, which is illustrated by an increase of the oscillation amplitude and a faster to appear vibration.

Then, the effects of proportional and non-proportional damping are investigated thanks to the same two studies as those considered in the previous section. In Fig. 8 it is clear that the addition of damping is synonymous with the decrease of the size of the limit cycle. To have a more visual representation, in Fig. 10 is plotted the sum of the maximum amplitudes -of displacement or velocity- taken on the degrees of freedom of the brake rod on the one hand, and on those of the axle on the other hand. The evolution with the amount of damping shows a decrease of a factor of 11 between the configuration with $0.5 \%$ of damping and the configuration with $3 \%$ of damping. $3 \%$ can be assumed to be a realistic value for mechanical systems using damping elements, and an amount of $0.5 \%$ of damping can also be possible in the worst case. But the damping coefficient can hardly reach zero because of the damping properties of the materials and the always dissipative contacts between the elements of the structure. So the advantages of using damping in order to reduce friction-induced vibrations appear here clearly.

In the second study the effects of non-proportional damping are examined. Fig. 9 illustrates the evolution of the limit cycle with the damping ratio, and Fig. 11 the evolution of the maximum amplitudes of the brake rod and the axle. It can be observed that an optimum is obtained as well for the displacements as for the velocities. The best configuration (the one for which the amplitudes are minimal) is given by a damping ratio near 1 , that confirms the results obtained in the section on the transient behavior. Considering a constant amount of damping, a change in the damping ratio can then result in oscillations almost two times bigger, which is absolutely not negligible. However, by placing damping elements only in the part of the system that vibrate the most, an asymmetry in the damping configuration can be obtained. This asymmetry could then be harmful because the mechanism could generate more vibration than the original undamped mechanism. 


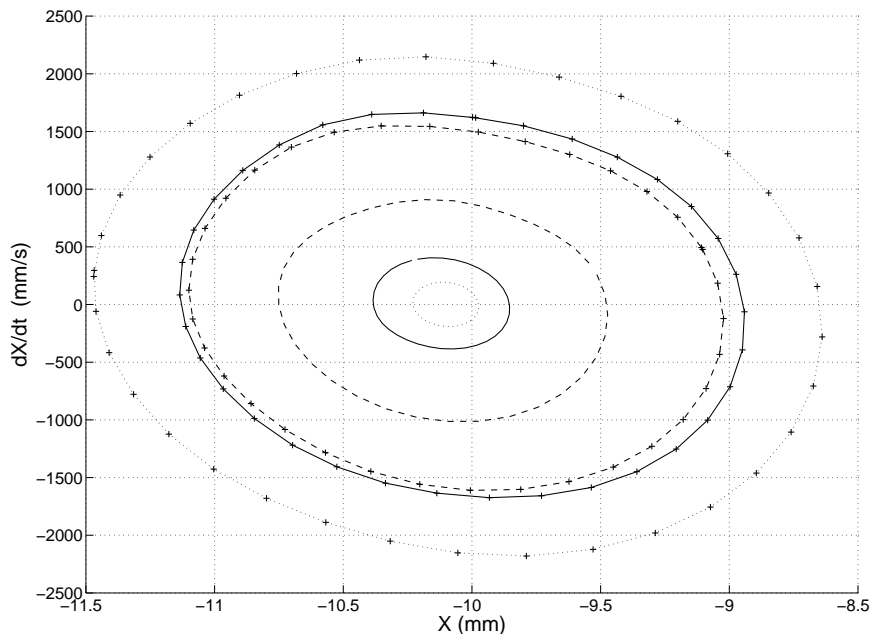

(a) End of the axle - horizontal

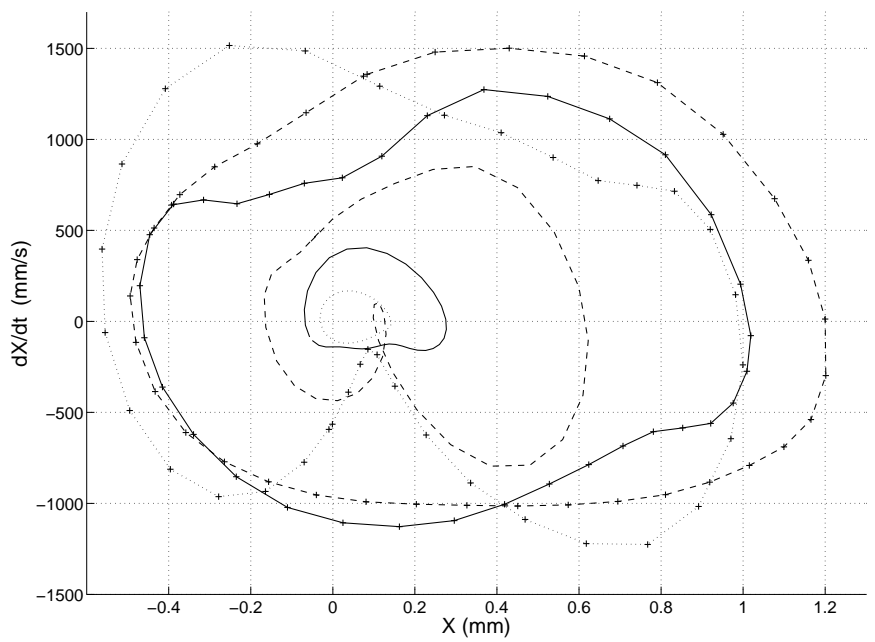

(c) Middle of the brake rod - horizontal

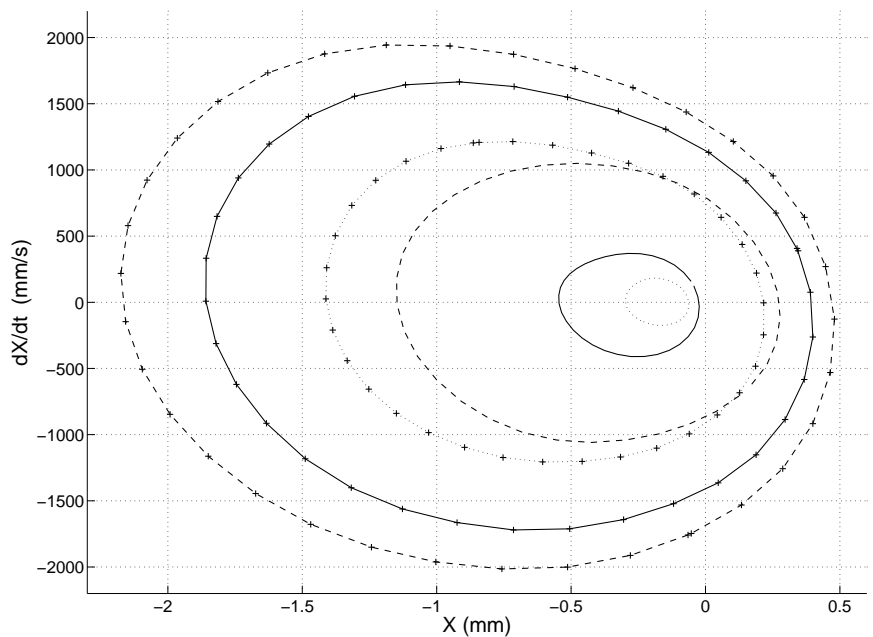

(b) End of the axle - vertical

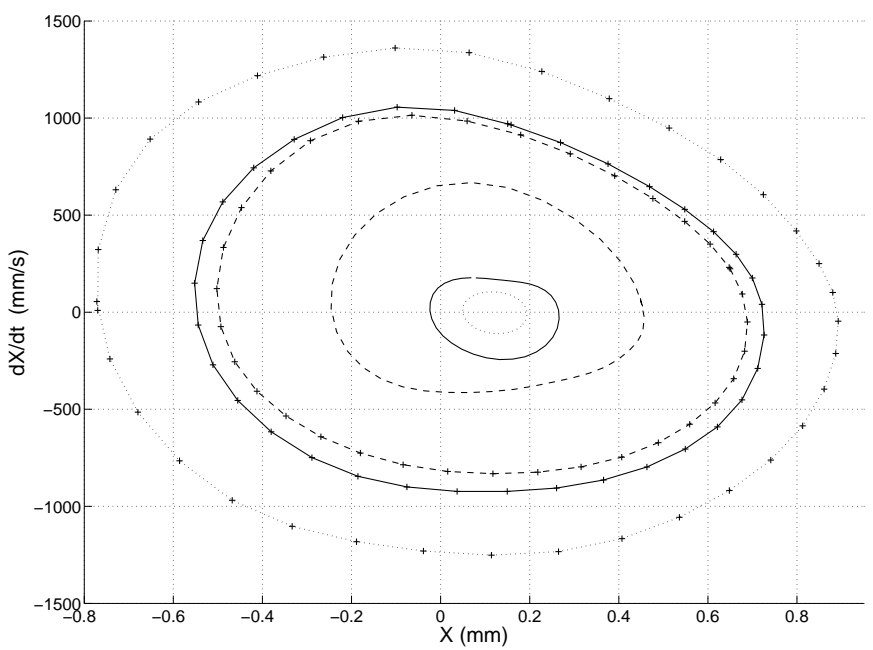

(d) Middle of the brake rod - vertical

Figure 7: Limit cycles - variations of $\mu\left(\cdots \mu / \mu_{\max }=0.05,--\mu / \mu_{\max }=0.075,--\mu / \mu_{\max }=0.1\right.$, $\left.\cdots+\cdots \mu / \mu_{\max }=0.125,-+-\mu / \mu_{\max }=0.15,-+-\mu / \mu_{\max }=0.175\right)$ 


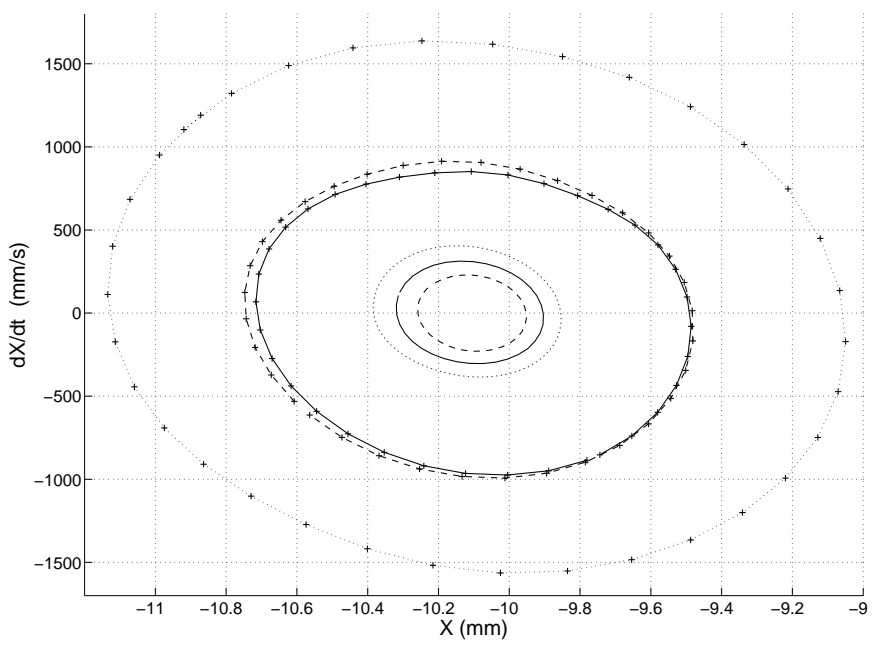

(a) End of the axle - horizontal

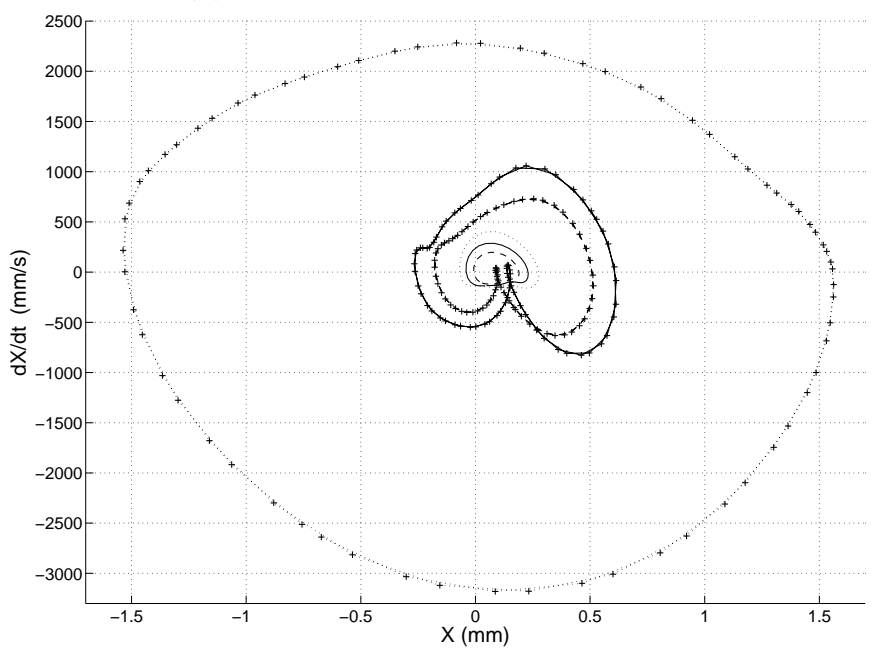

(c) Middle of the brake rod - horizontal

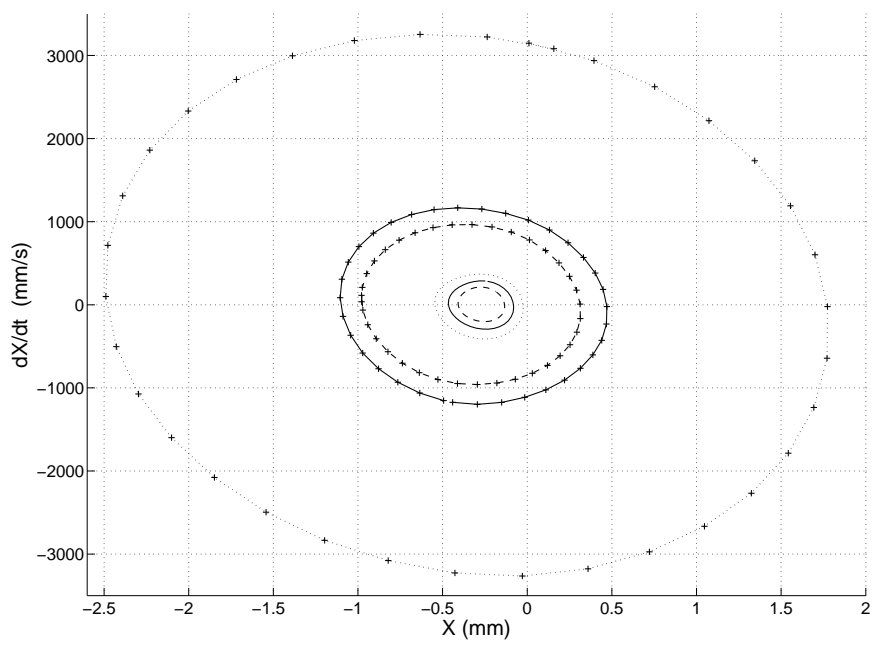

(b) End of the axle - vertical

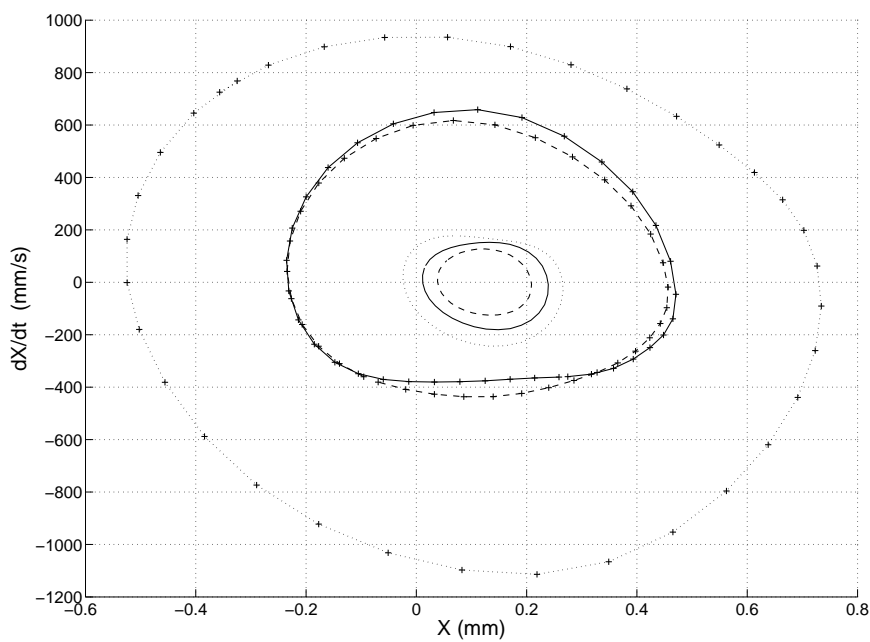

(d) Middle of the brake rod - vertical

Figure 8: Limit cycles - variations of proportional damping $(\cdots+\cdots \eta=0.005,-+-\eta=0.01,-+-\eta=$ $0.015, \cdots \eta=0.02,-\eta=0.025,--\eta=0.03)$ 


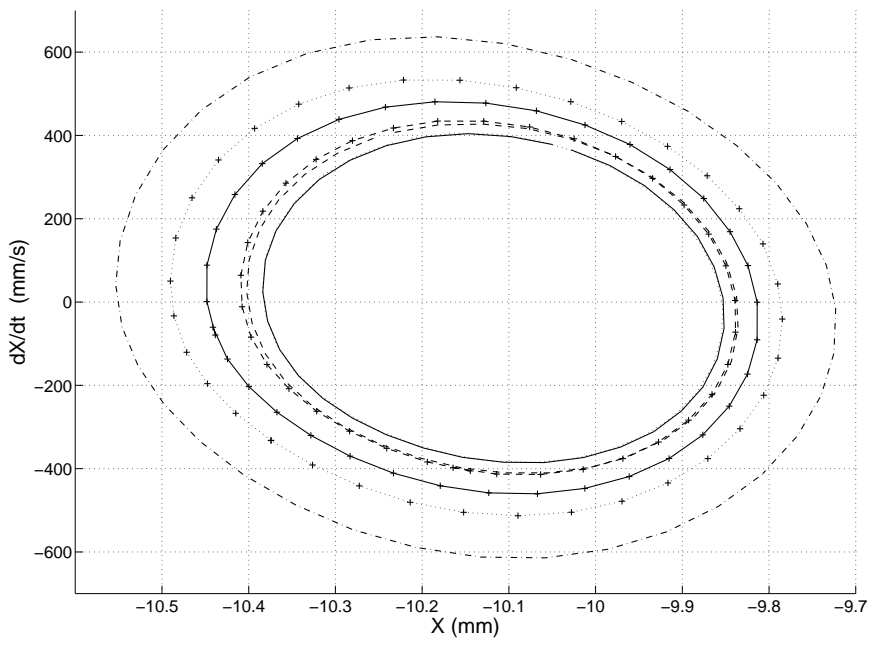

(a) End of the axle - horizontal

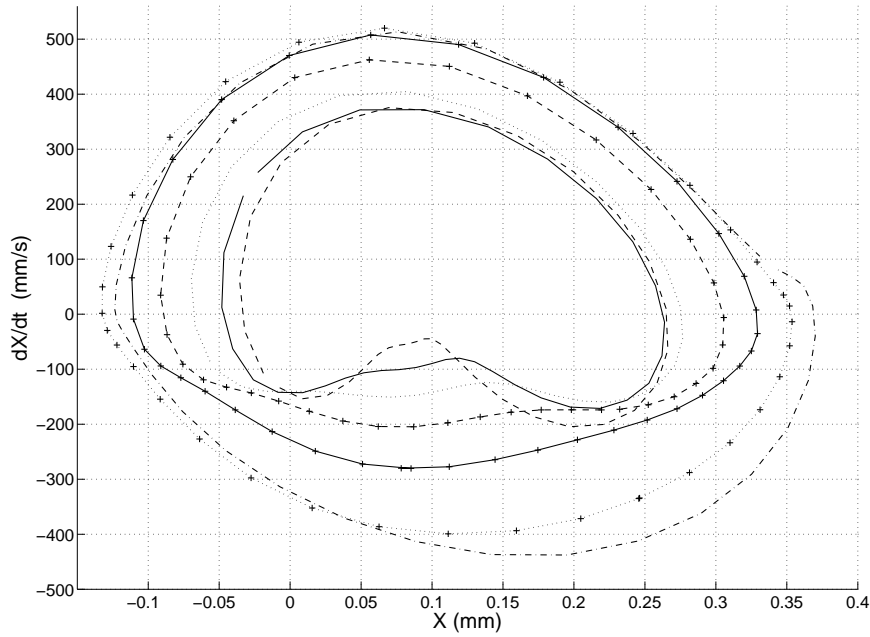

(c) Middle of the brake rod - horizontal

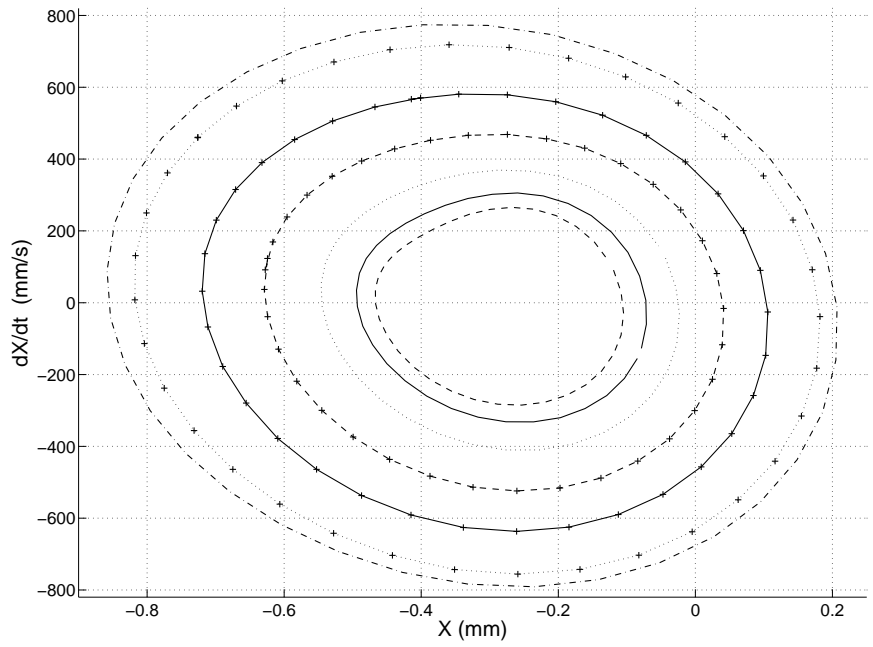

(b) End of the axle - vertical

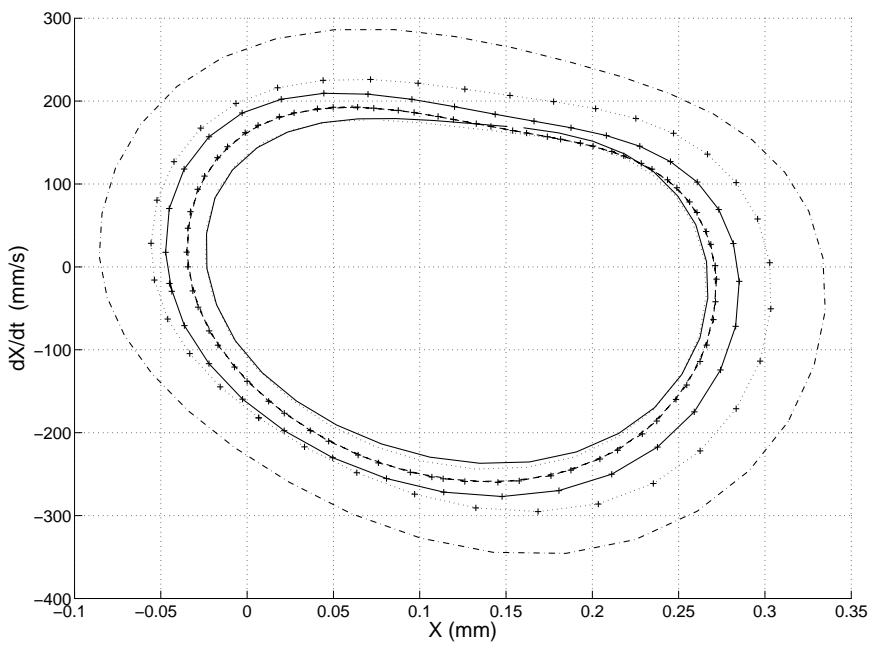

(d) Middle of the brake rod - vertical

Figure 9: Limit cycles - variations of non-proportional damping $\left(\cdots+\cdots \eta_{1} / \eta_{2}=0.14,-+-\eta_{1} / \eta_{2}=0.33\right.$, $\left.-+-\eta_{1} / \eta_{2}=0.6, \cdots \eta_{1} / \eta_{2}=1,-\eta_{1} / \eta_{2}=1.67,--\eta_{1} / \eta_{2}=3,-\cdot-\cdot \eta_{1} / \eta_{2}=7\right)$ 


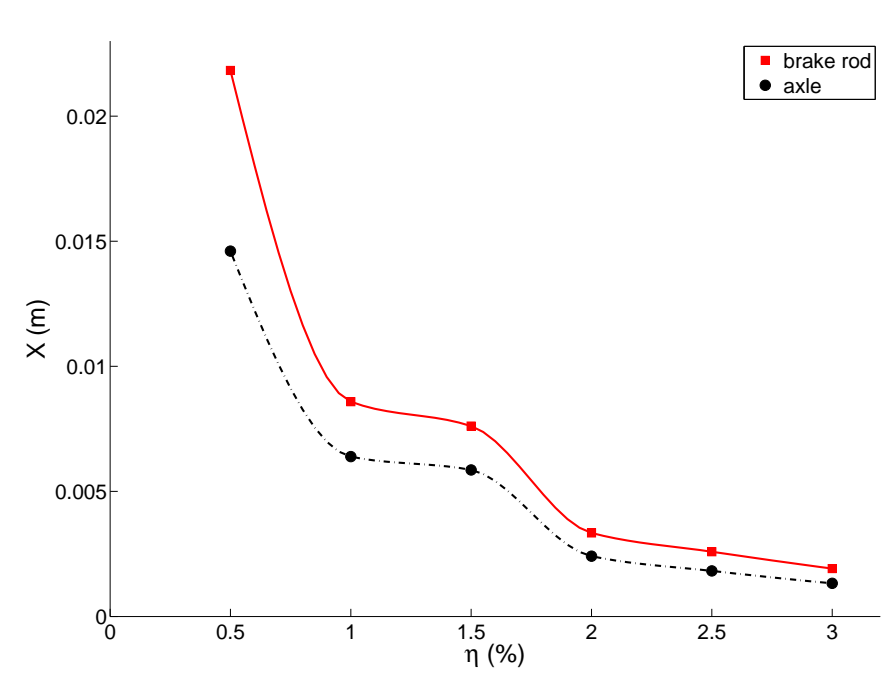

(a) Displacement

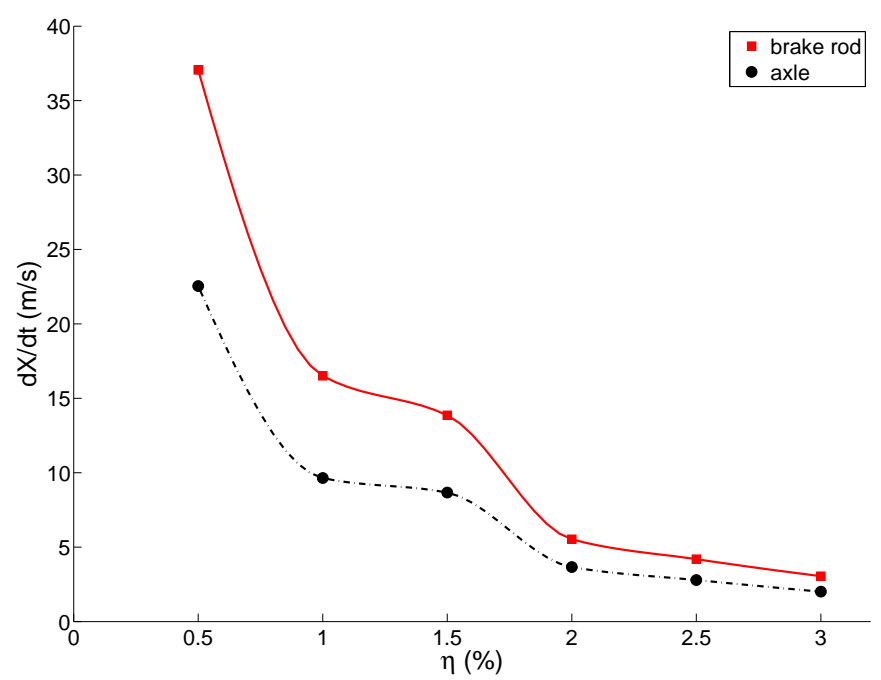

(b) Velocity

Figure 10: Vertical displacement of the axle - proportional damping

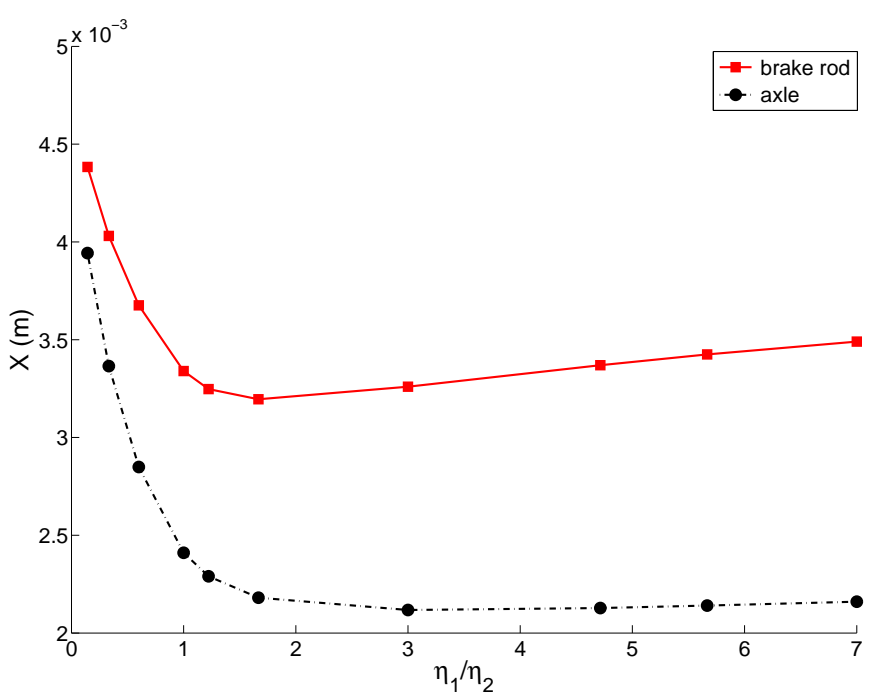

(a) Displacement

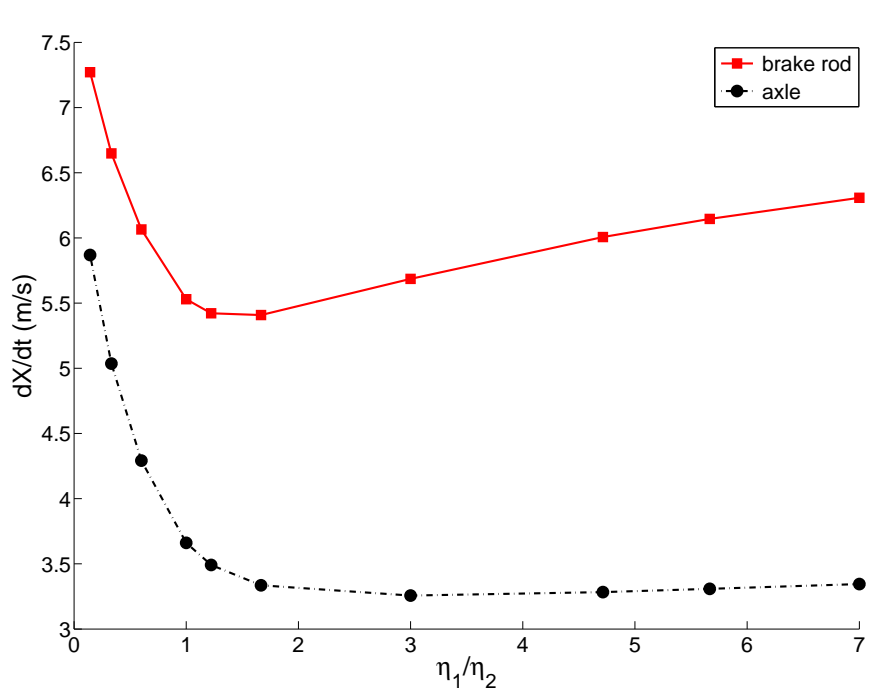

(b) Velocity

Figure 11: Vertical displacement of the axle - non-proportional damping 


\section{Summary and conclusions}

A nonlinear model has been developed in [1] for the stability analysis of mode-coupling instabilities induced by friction of an aircraft braking system. In this paper, the same model is used to evaluate the effects on the time-history response of the brake friction coefficient and the amount and ratio of damping. The first conclusion is that increasing the amount of damping leads to a vibration with a smaller amplitude and slower to develop. That points out the fact that damping can be very efficient to reduce or eliminate friction-induced vibrations. However, precautions have to be taken to avoid a misuse of damping. While in [1] it is shown that for this mechanism proportional damping is the best configuration for the stability of the system, the study presented leads to the same result about the dynamical behavior. At first sight it is not obvious for a nonlinear system because stability is obtained with linear approximation. Even if it may not be easy to design a system with an optimum damping distribution, in the light of the conclusions of this study, adding damping elements only in the part of the system that vibrate the most is an approach to avoid. In addition, the study shows that there exists a simple relation between the speed of increase of the vibration and the real part of the mode becoming unstable. Thus, the results of a stability analysis can be used in terms of temporal behavior, that is very interesting when time-history simulations are often time-consuming.

\section{References}

[1] Chevillot, F., Sinou, J.-J., Mazet, G.-B., Hardouin, N., and Jézéquel, L., 2008. "The destabilization paradox applied to friction-induced vibrations in an aircraft braking system". Archive of Applied Mechanics, 78 (12), pp. 949-963.

[2] Ziegler, H., 1952. "Die stabilitätskrieterien der elastomechanik”. Ingenieur-Archiv, 20, pp. 49-56.

[3] Bolotin, V. V., 1963. Non-conservative problems of the theory of elastic stability. Pergamon Press, Oxford.

[4] Bolotin, V. V., Grishko, A. A., Panov, M. Yu., 2002. Effect of damping on the postcritical behaviour of autonomous non-conservative systems. International Journal of Nonlinear Mechanics, 37, pp. 11631179.

[5] Kounadis, A. N., 1992. "On the paradox of the destabilizing effect of damping in nonconservative systems”. International Journal of Nonlinear Mechanics, 27, pp. 597-609.

[6] O’Reilly, O. M., Malhotra, N. K., and Namachchivaya, N. S., 1996. "Some aspects of destabilization in reversible dynamical systems with application to follower forces". Nonlinear Dynamics, 10 (1), pp. 63-87.

[7] Kirillov, O. N., and Seyranian, A. P., 2004. "Stabilization and destabilization of a circulatory system by small velocity-dependent forces". Journal of Sound and Vibration, 283, pp. 787-800.

[8] Herrmann, G., 1967. "Stability of equilibrium of elastic systems subjected to nonconservative forces". Applied Mechanical Reviews, 20 (2), pp. 103-108. 
[9] Walker, J. A., 1973. "A note on stabilizing damping configurations for linear non-conservative systems”. International Journal of Solids and Structures, 9 (12), pp. 1543-1545.

[10] Gallina, P., and Trevisani, A., 2003. "On the stabilizing and destabilizing effects of damping in wood cutting machines”. International Journal of Machine Tools and Manufacture, 43 (9), p. 955-964.

[11] Kirillov, O. N., and Seyranian, A. O., 2005. "The effect of small internal and external damping on the stability of distributed non-conservative systems". Journal of Applied Mathematics and Mechanics, 69 (4), pp. 529-552.

[12] Sinou, J.-J., and Jézéquel, L., 2007. "Mode coupling instability in friction induced vibrations and its dependency on system parameters including damping". European Journal of Mechanics - A/Solids, 26 (1), p. 106-122.

[13] Fritz, G., Sinou, J.-J., Duffal, J.-M., and Jézéquel, L., 2007. "Effects of damping on brake squeal coalescence patterns - application on a finite element model". Mechanics Research Communications, 34 (2), pp. 181-190.

[14] Hervé, B., Sinou, J.-J., Mahé, H., and Jézéquel, L., 2007. "Analysis of squeal noise and mode coupling instabilities including damping and gyroscopic effects". European Journal of Mechanics - A/Solids, 27 (2), pp. 141-160.

[15] Sinou, J.-J., Fritz, G., and Jézéquel, L., 2007. "The role of damping and definition of the robust damping factor (rd-factor) for a self-exciting mechanism with constant friction". Journal of Vibration and Acoustics, 129 (3), pp. 297-306. 